\title{
Capillary damping of inviscid surface waves in a circular cylinder
}

\author{
R. KID A MBI $\dagger$ \\ Computational \& Theoretical Fluid Dynamics Division, \\ National Aerospace Laboratories, Bangalore 560017, India
}

(Received 11 November 2008 and in revised form 7 January 2009)

We consider the effect of a wetting condition at the moving contact line on the frequency and damping of surface waves on an inviscid liquid in a circular cylinder. The velocity potential $\phi$ and the free surface elevation $\eta$ are sought as complex eigenfunction expansions. The $\phi$ eigenvalues are the classical ones whereas the $\eta$ eigenvalues are unknown and have to be computed so as to satisfy the wetting condition on the contact line and the other free surface conditions - these turn out to be complex in general. A projection of the latter conditions on to an appropriate basis leads to an eigenvalue problem, for the complex frequency $\Omega$, which has to be solved iteratively with the wetting condition. The variation of $\Omega$ with liquid depth $h$, Bond number $B o$, capillary coefficient $\lambda$ and static contact angle $\theta_{c 0}$ is explored for the $(1,0),(2,0),(0,1),(3,0)$ and $(4,0)$ modes. The damping vanishes for $\lambda=0$ (pinned-end edge condition) and $\lambda=\infty$ (free-end edge condition) with a maximum in the interior while the frequency decreases with increasing $\lambda$, approaching limiting values at the endpoints. A comparison with the analytic results of Miles (J. Fluid Mech., vol. 222, 1991, p. 197) for the no-meniscus case and the experimental results of Cocciaro, Faetti, \& Festa (J. Fluid Mech., vol. 246, 1993, p. 43), where a meniscus is present, is good. The study provides a simple procedure for calculating the inviscid capillary damping associated with the moving contact line in a circular cylinder of finite depth with meniscus effects also being considered.

\section{Introduction}

The damping of surface waves has been studied for at least 300 years. The main sources of damping are three: (i) viscous damping in the wall boundary layers and the bulk, (ii) capillary damping due to meniscus effects and the moving contact line and (iii) surface contamination effects. Early researches focused on unbounded domains and as such took into account only viscous damping in the bulk. For the last 100 years, surface contaminant effects have also been recognized; Lamb (1895) has a mathematical analysis of the effect of surface contamination on the damping. However, it has only been in the last 50 years that damping in finite spaces like containers began to be extensively studied. With these studies, the importance of capillary sources of damping like the region surrounding the contact line have begun to be appreciated. With the advent of spaceflights and the need to study fluid behaviour in microgravity conditions, meniscus effects on surface wave damping are being studied.

$\dagger$ Email address for correspondence: kidambi@ctfd.cmmacs.ernet.in 
Even though this subject has been studied for so long, many basic facts about it continue to be unknown or unclear. One longstanding mystery has been the discrepancy between experimental and estimated results for the damping rates; this could be sometimes as high as an order of magnitude. For example, it was shown only relatively recently (Martel, Nicolás \& Vega 1998) that the viscous damping in the bulk contributes at the same order as the damping in the wall boundary layers a fact that was missed by previous studies like Henderson \& Miles (1994); this has helped improve the agreement between theory and experiment at least when the contact line is pinned. The situation is much more complicated when the contact line moves. Even the behaviour of the static contact angle is not as straightforward as the Young-Laplace equation would have it; in many cases, it has been shown to be nonunique with a range of angles possible (capillary hysteresis). In such cases, the contact line would continue to be pinned till this range of angles is exceeded. Another vexing problem is that of the modelling of a moving contact line. An application of the noslip boundary condition at this line leads to a non-integrable stress singularity; various ad hoc remedies have been suggested and used but a fundamental understanding is still lacking.

Careful experiments under various conditions and meticulous analysis especially for essentially inviscid waves, i.e. at high Reynolds number, have contributed significantly to an understanding of surface wave damping in containers. Early experiments (Case \& Parkinson 1957; Keulegan 1959; Van Dorn 1966) were not very clear about the conditions obtaining at the contact line. Some recent studies (Cocciaro, Faetti \& Festa 1993; Howell et al. 2000; Jiang, Perlin \& Schultz 2004) have carefully measured and investigated the dynamic contact line and angle behaviours. Thus, we have two regimes reported in Cocciaro et al. (1993): a low-amplitude linear regime where the contact line is pinned and a higher amplitude nonlinear regime where the frequency varies with the amplitude of the contact line motion. Howell et al. (2000) is an extensive study of the frequency and damping rates of the lowest modes where the contact line is pinned throughout. The pinned contact line was first introduced by Benjamin \& Scott (1979) in order to explain certain experimental results; in these cases, the contact line remains at rest even when the free surface oscillates.

Modern theoretical studies have mostly been asymptotic analyses by Miles (1967, 1990), Hocking (1987) and Martel et al. (1998). The first two deal with the moving contact line but without really addressing the singularity there. The last one is for a pinned contact line but without considering the effects of a meniscus. Several eigenvalue formulations of various aspects of the problem have appeared as well: Nicolás (2002) studies viscous damping with a pinned contact line and flat interface; Nicolás (2005) considers the meniscus effect on the inviscid frequencies; Shankar (2007) calculates the frequencies of highly curved interfaces with pinned contact lines; and Kidambi (2009) studies a problem similar to Nicolás (2002) but with the meniscus additionally taken into account.

The boundary conditions at the contact line are known to affect the frequency and damping rate of surface waves. The classical boundary condition is of a contact line moving freely and a dynamic contact angle fixed at $90^{\circ}$. However, in reality, even the static contact angle $\theta_{c 0}$ is seldom $90^{\circ}$ and in fact may not have a unique value. The dynamic contact line and angle are capable of complex behaviours ranging from a pinned contact line to a moving contact line whose velocity $v_{C L}$ and the dynamic contact angle may be related. Miles (1967) was the first to propose a relation between the two. Based on experimental results (e.g. Ablett 1923), he assumed the existence of two regimes of motion, depending on whether the contact line velocity is larger 
than a critical value $v_{c}$. For $v_{C L}<v_{c}, \theta_{c}$ changes as a linear function of the contact line velocity while for $v_{C L}>v_{c}, \theta_{c}$ becomes independent of $v_{C L}$ and changes abruptly from $\theta_{R}$ for the receding contact line to $\theta_{a}$ for the advancing one. This implies the action of a Coulomb-like friction force $F=T\left[\cos \theta_{c}-\cos \theta_{c 0}\right]$ on the contact line. $\theta_{c}$ is the velocity-dependent dynamic contact angle. For $v_{C L}<v_{c}$, the case that we will be concerned with here, Miles assumed a contact line condition

$$
\frac{\partial \eta}{\partial t}=\lambda\left(\cos \theta_{c}-\cos \theta_{c 0}\right) \text {. }
$$

$\theta_{c 0}$ is assumed to be unique (no capillary hysteresis). Under the assumptions of $\theta_{c 0}=90^{\circ}$ and $\theta_{c}-\theta_{c 0} \ll 1$, the above condition reduces to the so-called wetting condition proposed by Hocking (1987). In this paper, we will use the terms 'Miles' condition' and 'Miles' wetting condition' to refer to (1.1) and its linearized version (2.1f), introduced in the next section. Note that the two extremes $\lambda=0$ and $\lambda=\infty$ give the pinned and free-end contact lines, respectively. For $\lambda=0$, since the contact line is pinned, the friction force does not work. Hence, the damping is zero in this case. For $\lambda=\infty$, the dynamic contact angle can be inferred to be a constant as the contact line moves with a finite velocity. Hence the Coulomb-like friction force is zero in this case and so is the damping.

There has been no study till now that deals with all aspects of the problem viscosity, capillarity, moving contact line - at once; the present one is no different. The modelling of a viscous moving contact line remains a problem for the future and will not be addressed here. We focus instead on the effect of capillarity on the frequency and damping of inviscid surface waves. Capillary damping is an important source of wave damping and can at times exceed viscous damping by an order of magnitude. The work being reported here is related to Hocking (1987), Nicolás (2005) and Shankar (2007). Hocking (1987) considers capillary damping in a rectangular geometry of infinite depth. Viscous effects are included through a boundary layer analysis but the analysis does not account for the meniscus and is restricted to a flat interface. This work is, in some sense, a generalization of Hocking to include meniscus effects and finite depth in a cylindrical geometry; viscous effects are excluded however. In a more straightforward sense, this paper is a generalization of Nicolás (2005) and Shankar (2007), which considered only $\lambda=0, \infty$ to the more complex Miles contact line condition. In this paper, a general eigenvalue problem for the complex frequency is formulated and solved for the effect of the Miles' wetting condition on inviscid capillary-gravity waves in a circular cylinder; there is no limitation on the Bond number or contact angle $\theta_{c 0}$. The two limiting cases $\lambda=0$ and $\lambda=\infty$ recover earlier known results for these cases. For other $\lambda$, we have a direct calculation of the capillary damping as well as the frequency for the first time.

The paper is organized as follows. Section 2 presents the governing equations and boundary conditions, $\S 3$ presents an eigenvalue formulation and solution of the problem where the natural eigenfunctions of the problem are used and $\S 4$ presents results for a range of capillary coefficient $\lambda$, liquid depth $h$, Bond number $B o$ and contact angle $\theta_{c 0}$ for the lowest modes. Comparisons with previous analytic results of Miles (1990) and experimental results of Cocciaro et al. (1993) are also presented.

\section{Governing equations}

We consider the small oscillations of inviscid liquid filling a circular cylindrical container of radius $R$ (figure 1). Scaling lengths by $R$ and time by $\sqrt{R / g}$, and 

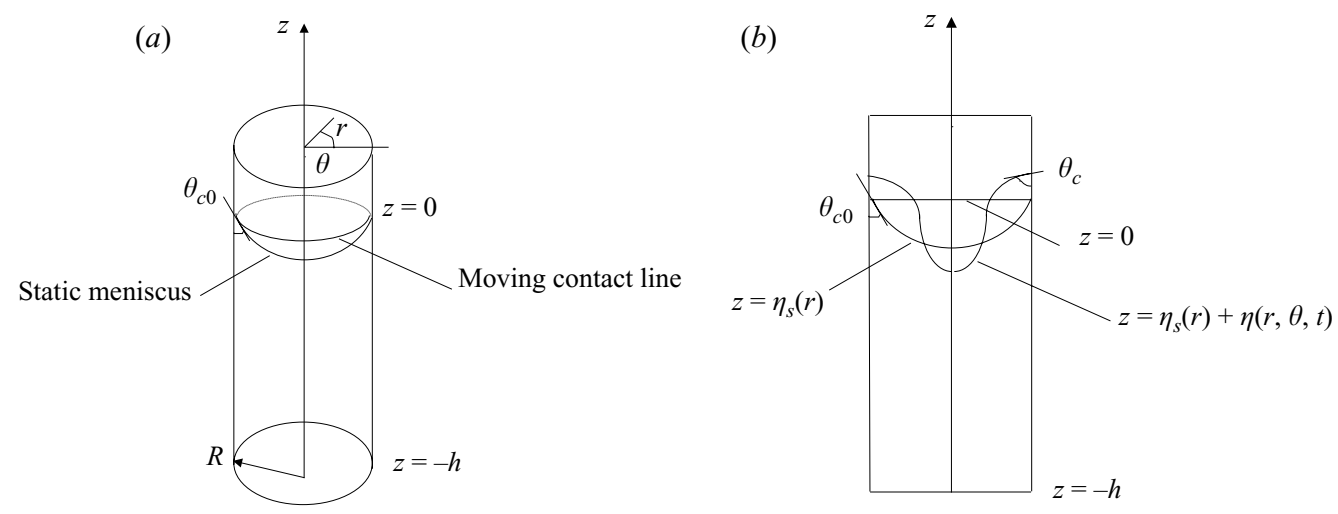

FIGURE 1. Geometry for oscillations of a free surface in a circular cylinder of radius $R$. The liquid is of depth $h$ and the contact line can slide on the vertical wall.

linearizing around the rest state, we have

$$
\begin{gathered}
\nabla^{2} \phi=0, \quad \phi_{r}(r=1, \theta, z, t)=0, \quad \phi_{z}(r, \theta, z=-h, t)=0, \\
\Omega \eta+\eta_{s}^{\prime} \phi_{r}=\phi_{z}, \quad \Omega \phi+\eta-\frac{1}{B o} \kappa=0 \quad \text { on } \quad z=\eta_{s}(r), \\
\Omega \eta=\lambda \eta^{\prime} /\left(1+\eta_{s}^{\prime 2}\right)^{3 / 2} \quad \text { on } \quad r=1 \quad \text { and } \int_{0}^{2 \pi} \int_{0}^{1} r \eta(r, \theta) \mathrm{d} r \mathrm{~d} \theta=0,
\end{gathered}
$$

where an oscillatory time dependence, with possibly complex frequency $\Omega$, has already been assumed. $\phi$ is the velocity potential governing the flow, $\eta$ is the free surface elevation measured from the static meniscus $z=\eta_{s}(r), B o=\rho g R^{2} / \sigma$ is the Bond number and $\lambda$ is the capillary coefficient. $\kappa$ is the first-order perturbation to the curvature and is given by (Shankar 2007)

$$
\kappa(r, \theta, t)=p(r) \eta_{r}+q(r) \eta_{r r}+\bar{r}(r) \eta_{\theta \theta},
$$

where

$$
p(r)=\frac{\left(1+3 \eta_{s}^{\prime 2}\right)}{r\left(1+\eta_{s}^{\prime 2}\right)}, \quad q(r)=\frac{1}{\left(1+\eta_{s}^{\prime 2}\right)^{3 / 2}}, \quad \bar{r}(r)=\frac{1}{r^{2}\left(1+\eta_{s}^{\prime 2}\right)^{1 / 2}} .
$$

Equations $(2.1 a-c)$ are standard conditions for potential flow, $(2.1 d, e)$ are the standard kinematic and dynamic conditions on the free surface $z=\eta_{s}(r)+\eta(r, \theta, t)$ (Johnson 1997), $(2.1 f)$ is the Miles' wetting condition on the moving contact line and the conservation of fluid volume is given by $(2.1 \mathrm{~g})$. Note that, under linearization, $(2.1 \mathrm{~d}, e)$ are applied on the static meniscus $z=\eta_{s}(r)$. Using

$$
\cos \left(\theta_{c}\right)-\cos \left(\theta_{c 0}\right)=\frac{\eta_{s}^{\prime}+\eta^{\prime}}{\sqrt{1+\left(\eta_{s}^{\prime}+\eta^{\prime}\right)^{2}}}-\frac{\eta_{s}^{\prime}}{\sqrt{1+\eta_{s}^{\prime 2}}},
$$

(2.1f) is obtained by linearizing (1.1) for small $\eta^{\prime}$. The static meniscus $z=\eta_{s}(r)$ satisfies the differential equation

$$
\eta_{s}(r)=\frac{1}{B o} \kappa_{s}(r)+\zeta, \quad \text { with } \quad \kappa_{s}(r)=\frac{\eta_{s}^{\prime \prime}+\eta_{s}^{\prime}\left(1+\eta_{s}^{\prime 2}\right) / r}{\left(1+\eta_{s}^{\prime 2}\right)^{3 / 2}},
$$

where a prime denotes differentiation with respect to $r . \zeta$ is an unknown parameter that has to be found as a part of the solution and appears in $(2.2 a)$ because the 
meniscus is invariant under translations along the $z$-axis. In the current definition (figure 1), where $\eta_{s}(1)=0, \zeta$ is just the negative of the curvature of the static meniscus at the wall, scaled by the Bond number.

\section{Solution}

A harmonic function satisfying $(2.1 a-c)$ is easily found and $\phi$ is written as

$$
\phi(r, \theta, z)=\cos m \theta \sum_{n=1}^{N} b_{n} J_{m}\left(v_{n} r\right) \frac{\cosh v_{n}(z+h)}{\cosh v_{n} h},
$$

where $J_{m}$ is the Bessel function of first kind of order $m$ and $J_{m}^{\prime}\left(v_{n}\right)=0$. We denote the oscillatory modes by $(m, k)$, where $m$ denotes the number of azimuthal nodes (nodal diameters) and $k$ the number of nodes in the radial direction (nodal circles). Note that an infinite set of $\left(b_{n}, v_{n}\right)$ is associated with each $k$, which for the purposes of computation has been truncated to a set of dimension $N$, as in (3.1). We seek $\eta$ as

$$
\eta(r, \theta)=\cos m \theta \sum_{n=1}^{N} a_{n} J_{m}\left(\delta_{n} r\right),
$$

where the spatial eigenvalues $\delta_{n}$ are as yet unknown. Note that this representation satisfies the volume conservation condition $(2.1 \mathrm{~g})$ for the non-axisymmetric modes $m \neq 0$. Using this representation for $\eta$ in the Miles' wetting condition $(2.1 f)$, we obtain

$$
\sum_{n=1}^{N} a_{n}\left(\Omega J_{m}\left(\delta_{n}\right)-\hat{\lambda} \delta_{n} J_{m}^{\prime}\left(\delta_{n}\right)\right)=0,
$$

where $\hat{\lambda}=\lambda /\left(1+\eta_{s}^{\prime 2}\right)^{3 / 2}$. The static meniscus $z=\eta_{s}(r)$ is computed by solving $(2.2 a)$, which is a two-point boundary eigenvalue problem. The two boundary conditions are $\eta_{s}^{\prime}(1)=\cot \theta_{c 0}$ and $\eta_{s}^{\prime}(0)=0$. With the coordinates fixed such that $\eta(1)=0$ (pinned contact line), (2.2a) has solutions only for particular values of $\zeta$ which is an unknown parameter. The solution of $(2.2 a)$ is standard; we use a minimization procedure outlined in the Appendix. Now only $(2.1 d, e)$ remain to be satisfied. We employ a weighted residual method and require the inner product of these equations with the first $N$ members of a complete set of test functions to vanish. We choose the test functions as the set $\left\{J_{m}\left(\mu_{l} r\right), l=1,2, \ldots\right\}$, where the $\mu_{l}$ are the roots of $J_{m}$. We project the kinematic and dynamic free surface conditions $(2.1 d)$ and $(2.1 e)$ to get the set of $2 N$ equations

$$
\begin{gathered}
\Omega \sum_{n=1}^{N} a_{n} \beta_{n l}^{1}+\sum_{n=1}^{N} b_{n} \xi_{n l}^{1}=0, \\
\Omega \sum_{n=1}^{N} b_{n} \xi_{n l}^{2}+\sum_{n=1}^{N} a_{n} \beta_{n l}^{2}=0, \quad l=1, \ldots, N,
\end{gathered}
$$

with the integrals $\beta_{n l}^{1}, \ldots$ given by

$$
\begin{array}{r}
\beta_{n l}^{1}=\int_{0}^{1} r J_{m}\left(\delta_{n} r\right) J_{m}\left(\mu_{l} r\right) \mathrm{d} r, \quad \xi_{n l}^{2}=\int_{0}^{1} r J_{m}\left(v_{n} r\right) J_{m}\left(\mu_{l} r\right) \mathrm{d} r \\
\beta_{n l}^{2}=\left(1+\frac{\delta_{n}^{2}}{B o}\right) \beta_{n l}^{1}, \quad \xi_{n l}^{1}=v_{n} \tanh v_{n} h \xi_{n l}^{2} .
\end{array}
$$


Equations (3.4) become a matrix eigenvalue problem for $\Omega$ with a small twist in that $\delta_{n}, n=1, \ldots, N$ are not known a priori. Equations (3.3) and (3.4) have to be solved together iteratively. The numerical procedure consists of the following steps:

(a) Start with initial guesses for $\Omega$ and $\delta_{n}, n=1, \ldots, N$ as $\Omega_{i}$ and $\delta_{n i}$, respectively.

(b) Solve the eigenvalue problem (3.4) with $\delta_{n i}$ and obtain $\Omega_{f}$.

(c) Use $\Omega_{i}=\alpha_{0} \Omega_{i}+\left(1-\alpha_{0}\right) \Omega_{f}$, with $\alpha_{0} \in(0,1)$ in (3.3) to solve for $\delta_{n f}$.

(d) Repeat step (b) with $\delta_{n f}$.

(e) If $\operatorname{Re}\left\{\Omega_{f}-\Omega_{i}\right\}$ and $\operatorname{Im}\left\{\Omega_{f}-\Omega_{i}\right\}<\epsilon_{o}$ for a chosen tolerance, the calculation is done. If not, repeat $(c)$ and $(d)$ till convergence.

It is convenient to start the calculation with the classical free-end solution for $\lambda=\infty$, given by

$$
\Omega_{i}^{2}=-v_{n} \tanh v_{n} h\left(1+v_{n}^{2} / B o\right), \quad \delta_{n}=v_{n},
$$

with the $v_{n}$ given by $J_{m}^{\prime}\left(v_{n}\right)=0$. These values are then continued through lower values of $\lambda$ to $\lambda=0$. The procedure works well in general with convergence usually being achieved in under 10 iterations. Note that this procedure has to be repeated for the different modes, i.e. even though $N \Omega$ are obtained by solving (3.4), all these will not be the correct values as the iteration was performed with respect to only one of those temporal eigenvalues. In other words, each modal frequency has corresponding to it a different set of spatial eigenvalues. The axisymmetric case $m=0$ is more subtle. Now mass conservation is not automatic unlike in the non-axisymmetric case and has to be enforced. This is done by seeking

$$
\eta(r)=\sum_{i=1}^{N} a_{n}\left(J_{0}\left(\delta_{n} r\right)-A_{n} f(r)\right),
$$

where $f(r)$ is a conveniently chosen function and $A_{n}$ are constants such that the volume conservation condition $(2.1 \mathrm{~g})$ is satisfied. A convenient choice for $f(r)$ is $\left(1-r^{2}\right)^{2}$. Since $f(1)=f^{\prime}(1)=0$, the Miles' wetting condition (3.3) remains unchanged. The $A_{n}$ are given by

$$
A_{n}=\frac{\int_{0}^{1} r J_{0}\left(\delta_{n} r\right) \mathrm{d} r}{\int_{0}^{1} r f(r) \mathrm{d} r}=6 \int_{0}^{1} r J_{0}\left(\delta_{n} r\right) \mathrm{d} r .
$$

Using this new representation and the fact that a constant term has to be included in the expansion for $\phi$ (Shankar 2007) leads to a similar eigenvalue problem as for the non-axisymmetric modes.

\section{Results and discussion}

\subsection{Computation of the static meniscus}

The static menisci for all the Bond numbers and a variety of contact angles, for which computations have been performed, have been calculated as the first step in the calculation of $\Omega$. Up to 2000 eigenfunctions and 4800 minimization points were used in the meniscus calculations. The maximum error in the satisfaction of $(2.2 a)$ was of $O\left(10^{-6}\right)$.

\subsection{Limiting eigenvalue distributions}

We present corrections to the eigenvalues $\delta_{n}$ in the two limiting cases $\lambda \rightarrow \infty$ and $\lambda \rightarrow 0$. 


\subsection{1. $\lambda \rightarrow \infty$ (free-end edge condition)}

For $\lambda \rightarrow \infty$, let $\epsilon=1 / \lambda$ and seek

$$
\begin{array}{ll}
a_{n}=a_{n 0}+\epsilon a_{n 1}+\cdots, & b_{n}=b_{n 0}+\epsilon b_{n 1}+\cdots, \\
\Omega=\Omega_{0}+\epsilon \Omega_{1}+\cdots, & \delta_{n}=\delta_{n 0}+\epsilon \delta_{n 1}+\cdots .
\end{array}
$$

Inserting these expressions in (3.3) and (3.4) and equating powers of $\epsilon$, we have the equations at the lowest orders as

$O(1)$

$$
\begin{gathered}
J_{m}^{\prime}\left(\delta_{n 0}\right)=0, \quad \Omega_{0} \sum_{n=1}^{N} a_{n 0} \beta_{n l 0}^{1}+\sum_{n=1}^{N} b_{n 0} \xi_{n l}^{1}=0 \\
\Omega_{0} \sum_{n=1}^{N} b_{n 0} \xi_{n l}^{2}+\sum_{n=1}^{N} a_{n 0}\left(1+\frac{\delta_{n 0}^{2}}{B o}\right) \beta_{n l 0}^{1}, \quad \text { where } \quad \beta_{n l 0}^{1}=\int_{0}^{1} r J_{m}\left(\delta_{n 0} r\right) J_{m}\left(\mu_{l} r\right) \mathrm{d} r .
\end{gathered}
$$

$\underline{O(\epsilon)}$

$$
\begin{gathered}
\Omega_{0} J_{m}\left(\delta_{n 0}\right)-\frac{\delta_{n 0} \delta_{n 1} J_{m}^{\prime \prime}\left(\delta_{n 0}\right)+\delta_{n 1} J_{m}^{\prime}\left(\delta_{n 0}\right)}{\left(1+\eta_{s}^{\prime 2}\right)^{3 / 2}}=0, \\
\Omega_{0} \sum_{n=1}^{N}\left(a_{n 0} \beta_{n l 1}^{1}+a_{n 1} \beta_{n l 0}^{1}\right)+\Omega_{1} \sum_{n=1}^{N} a_{n 0} \beta_{n l 0}^{1}+\sum_{n=1}^{N} b_{n 1} \xi_{n l}^{1}=0, \\
\Omega_{0} \sum_{n=1}^{N} b_{n 1} \xi_{n l}^{2}+\Omega_{1} \sum_{n=1}^{N} b_{n 0} \xi_{n l}^{2}+\sum_{n=1}^{N} a_{n 1}\left[\beta_{n l 1}^{1}\left(1+\frac{\delta_{n 0}^{2}}{B o}\right)+2 \beta_{n l 0}^{1} \frac{\delta_{n 1}}{B o}\right]=0, \\
\text { where } \beta_{n l 1}^{1}=\delta_{n 1} \int_{0}^{1} r^{2} J_{m}^{\prime}\left(\delta_{n 0} r\right) J_{m}\left(\mu_{l} r\right) \mathrm{d} r .
\end{gathered}
$$

At $O(1)$, the solution is

$$
\delta_{n 0}=v_{n}, \quad \Omega_{0}^{2}=-v_{n} \tanh v_{n} h\left(1+\frac{v_{n}^{2}}{B o}\right),
$$

where we have used the fact that $\beta_{n l 0}^{1}=\xi_{n l}^{2}$. This is just the classical free-end edge solution. Note that at this order, the modes are uncoupled and each temporal eigenvalue $\Omega_{0}$ has a spatial eigenvalue $\delta_{0} \equiv \delta_{n 0}$ corresponding to it. However, at higher orders, each modal frequency $\Omega$ will have a set of $\delta_{n i}, i=1, \ldots, N$ corresponding to it.

At $O(\epsilon)$, the solution is given by

$$
\delta_{n 1}=\frac{\Omega_{0} J_{m}\left(\delta_{n 0}\right)}{\delta_{n 0} J_{m}^{\prime \prime}\left(\delta_{n 0}\right)}\left(1+\eta_{s}^{\prime 2}\right)^{3 / 2} .
$$

$\Omega_{1}$ cannot be obtained in closed form; it is obtained by solving the matrix eigenvalue problem at $O(\epsilon)$.

\subsection{2. $\lambda \rightarrow 0$ (pinned-end edge condition)}

For $\lambda \rightarrow 0$, letting $\epsilon=\lambda$ and following a similar procedure as above yields at the lowest orders 
$\delta$

\begin{tabular}{|c|c|c|}
\hline$\lambda$ & Analytic & Numerical \\
\hline 100 & $\begin{array}{l}1.8412-0.0106 \mathrm{i} \\
5.3314-0.0027 \mathrm{i} \\
8.5363-0.0016 \mathrm{i}\end{array}$ & $\begin{array}{l}1.8412-0.0106 \\
5.3314-0.0027 \\
8.5363-0.0016\end{array}$ \\
\hline 10 & $\begin{array}{l}1.8412-0.1062 \mathrm{i} \\
5.3314-0.0268 \mathrm{i} \\
8.5363-0.0164 \mathrm{i}\end{array}$ & $\begin{array}{l}1.8470-0.1058 \\
5.3316-0.0268 \\
8.5364-0.0164\end{array}$ \\
\hline 0.01 & $\begin{array}{c}3.8317-0.0244 \mathrm{i} \\
7.0156-0.0446 \mathrm{i} \\
10.173-0.0647 \mathrm{i}\end{array}$ & $\begin{array}{r}3.8315-0.02441 \\
7.0151-0.0447 \\
10.1728-0.0648\end{array}$ \\
\hline 0.001 & $\begin{array}{r}3.8317-0.0024 \mathrm{i} \\
7.0156-0.0045 \mathrm{i} \\
10.173-0.0065 \mathrm{i}\end{array}$ & $\begin{array}{r}3.8317-0.0024 \\
7.0156-0.0045 \\
10.1735-0.0065\end{array}$ \\
\hline
\end{tabular}

TABLE 1. The first three eigenvalues $\delta_{n}$ for the $(1,0)$ mode for a few large and small $\lambda$. Here $h=2, B o=100, \theta_{c 0}=90^{\circ}$. The comparison between the analytic and numerical values is very good.

$\underline{O(1)}$

$$
\begin{gathered}
J_{m}\left(\delta_{n 0}\right)=0, \quad \Omega_{0} \sum_{n=1}^{N} a_{n 0} \beta_{n l 0}^{1}+\sum_{n=1}^{N} b_{n 0} \xi_{n l}^{1}=0 \\
\Omega_{0} \sum_{n=1}^{N} b_{n 0} \xi_{n l}^{2}+\sum_{n=1}^{N} a_{n 0}\left(1+\frac{\delta_{n 0}^{2}}{B o}\right) \beta_{n l 0}^{1}, \quad \text { where } \quad \beta_{n l 0}^{1}=\int_{0}^{1} r J_{m}\left(\delta_{n 0} r\right) J_{m}\left(\mu_{l} r\right) \mathrm{d} r
\end{gathered}
$$

$\underline{O(\epsilon)}$

$$
\begin{gathered}
\Omega_{1} J_{m}\left(\delta_{n 0}\right)+\left(\Omega_{0} \delta_{n 1}-\frac{\delta_{n 0}}{\left(1+\eta_{s}^{\prime}\right)^{3 / 2}}\right) J_{m}^{\prime}\left(\delta_{n 0}\right)=0, \\
\Omega_{0} \sum_{n=1}^{N}\left(a_{n 0} \beta_{n l 1}^{1}+a_{n 1} \beta_{n l 0}^{1}\right)+\Omega_{1} \sum_{n=1}^{N} a_{n 0} \beta_{n l 0}^{1}+\sum_{n=1}^{N} b_{n 1} \xi_{n l}^{1}=0, \\
\Omega_{0} \sum_{n=1}^{N} b_{n 1} \xi_{n l}^{2}+\Omega_{1} \sum_{n=1}^{N} b_{n 0} \xi_{n l}^{2}+\sum_{n=1}^{N} a_{n 1}\left[\beta_{n l 1}^{1}\left(1+\frac{\delta_{n 0}^{2}}{B o}\right)+2 \beta_{n l 0}^{1} \frac{\delta_{n 1}}{B o}\right]=0, \\
\text { where } \beta_{n l 1}^{1}=\delta_{n 1} \int_{0}^{1} r^{2} J_{m}^{\prime}\left(\delta_{n 0} r\right) J_{m}\left(\mu_{l} r\right) \mathrm{d} r .
\end{gathered}
$$

At $O(1), \delta_{n 0}$ are just the roots of the Bessel function $J_{m}$. However, $\Omega_{0}$ cannot be obtained in closed form as in the free-end edge case; the matrix eigenvalue problem at this order has to be solved.

At $O(\epsilon)$, the solution is given by $\delta_{n 1}=\delta_{n 0} /\left(\Omega_{0}\left(1+\eta_{s}^{\prime 2}\right)^{3 / 2}\right)$. Again, $\Omega_{1}$ cannot be obtained in closed form; it is obtained by solving the matrix eigenvalue problem at $O(\epsilon)$.

Table 1 shows the analytic and numerically computed values of $\delta_{n}$ for large and small $\lambda$ for $B o=100, h=2$. As can be seen, the two-term analytic solutions are fairly 


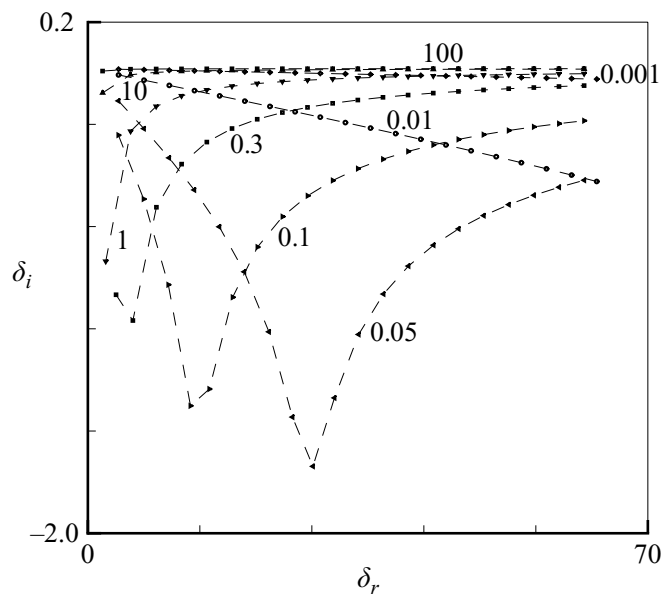

FiguRE 2. Spatial eigenvalues $\delta$ as a function of the capillary coefficient $\lambda$ for the $(1,0)$ mode. Here $B o=100$ and $h=2$.

\begin{tabular}{|c|c|c|c|c|}
\hline \multirow[b]{2}{*}{$\lambda$} & \multicolumn{2}{|c|}{$-\Omega_{r}$} & \multicolumn{2}{|c|}{$\Omega_{i}$} \\
\hline & $N=20$ & $N=40$ & $N=20$ & $N=40$ \\
\hline 100 & $2.6083 \times 10^{-5}$ & $2.6083 \times 10^{-5}$ & 1.358 & 1.358 \\
\hline 10 & $2.6082 \times 10^{-4}$ & $2.6082 \times 10^{-4}$ & 1.358 & 1.358 \\
\hline 1 & $2.6029 \times 10^{-3}$ & $2.6030 \times 10^{-3}$ & 1.358 & 1.358 \\
\hline 0.1 & $2.1603 \times 10^{-2}$ & $2.1690 \times 10^{-2}$ & 1.369 & 1.368 \\
\hline 0.01 & $8.2281 \times 10^{-3}$ & $1.0769 \times 10^{-2}$ & 1.411 & 1.414 \\
\hline 0.001 & $8.1414 \times 10^{-4}$ & $1.0357 \times 10^{-3}$ & 1.411 & 1.415 \\
\hline
\end{tabular}

TABLE 2. Convergence study for the frequency and damping rate of the $(1,0)$ mode. Twenty and 40 modes have been used. Here $h=2, B o=1000$.

accurate up to $\lambda=0.01$ and down to $\lambda=10$. Numerically computed $\delta_{n}$ for various $\lambda$ are shown in figure 2.

\subsection{Numerical results}

We start off by presenting a convergence study in table 2 ; the calculations with $N=20$ and $N=40$ modes show that 20 modes are sufficient for convergence to three decimal places down till $\lambda=0.1$. However, more modes will be required as $\lambda \rightarrow 0$. We have used 20 modes in all the calculations which have been presented here. In most of the calculations presented here, the bottom wall boundary conditions are satisfied to $O\left(10^{-10}\right)$ and the convergence criterion for the iterative procedure of $\S 3, \epsilon$ has been fixed at $10^{-6}$.

Miles (1967) has shown that the capillary damping is proportional to the modal frequency when $v_{C L}<v_{c}$, where $v_{C L}$ was assumed to be the same as that given by the free-end edge conditions. Miles (1991) showed the damping to be proportional (inversely proportional) to $\lambda$ for $\lambda \ll 1(\gg 1)$. This means that the capillary damping rate is higher for the higher modes and for lower Bond numbers. This is borne out by the results presented in figures 3 and 4 . We present the variation of $\Omega_{r}$ and $\Omega_{i}$ with the capillary coefficient $\lambda$ in figure 3 . These results are for the no-meniscus case of $\theta_{c 0}=90^{\circ}$. For $\lambda \gg 1,-\Omega_{r}$ increases with decreasing $B o$. It exhibits a maximum for $\lambda>0$ and goes to zero for $\lambda \rightarrow 0, \infty$ as expected. The location of maximum damping 
(a)

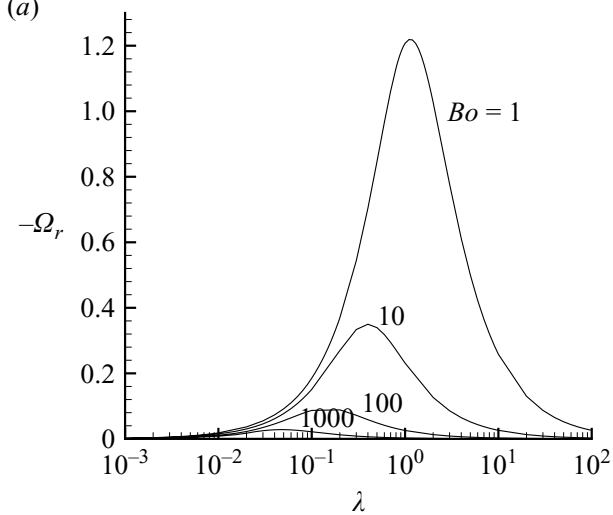

(b)

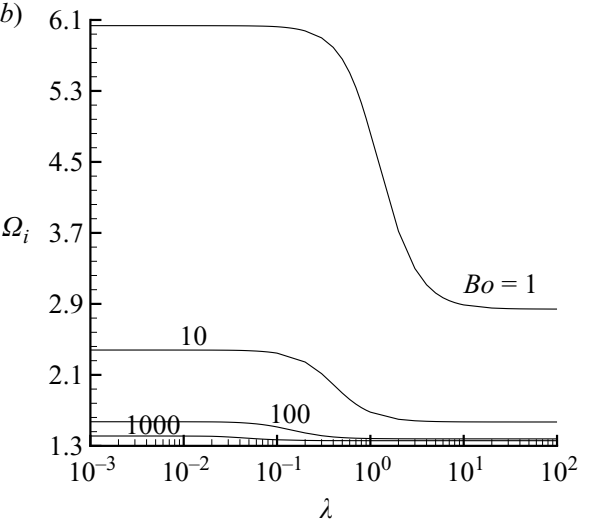

FIGURE 3. Variation of the $(a)$ damping rates and $(b)$ frequencies of the $(1,0)$ mode with $\lambda$. In $(a)$, the curves from top to bottom correspond to $B o=1,10,100$ and 1000, respectively. Here $h=2$.
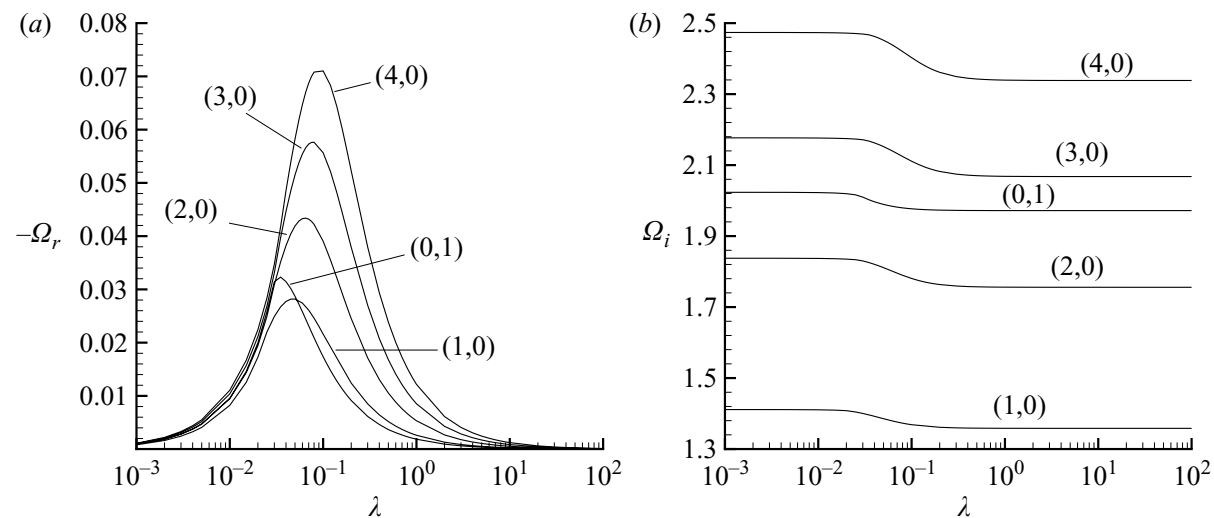

Figure 4. Variation of the $(a)$ damping rates and $(b)$ frequencies of the $(0,1),(1,0),(2,0),(3,0)$ and $(4,0)$ modes with $\lambda$. Here $h=2, B o=100$.

shifts to lower $\lambda$ for increasing $B o$. According to the boundary layer approximation in Miles (1991), the maximum damping rate is $-\Omega_{r M}=1.42 / \sqrt{B o}$ and the maximum location is at $\lambda_{M}=1 / \sqrt{B o}$. As regards the frequency, the pinned contact line frequency $\Omega_{i}^{p}(\lambda=0)$ is larger than the free contact line frequency $\Omega_{i}^{f}(\lambda=\infty)$. The two values are already attained for $\lambda=0.1$ and 10 , respectively. A large percentage of the frequency change from $\Omega_{i}^{p}$ to $\Omega_{i}^{f}$ occurs over approximately a two-decadal range of $\lambda$. This range is a function of $B o$ and shifts towards lower $\lambda$ as $B o$ increases. $\Omega_{i}^{p}-\Omega_{i}^{f}$ increases with decreasing $B o$.

Figure 4 shows similar results for the five lowest modes for $h=2$ and $B o=100$. The frequencies and damping rates show the same trends as before with the damping rates of the higher modes being larger than those of the lower ones over the entire $\lambda$ range. The exception is the $(0,1)$ mode whose damping rate is higher than that of the $(1,0)$ mode for $\lambda<0.05$ and lower for values above.

Figure 5 shows the variations with $\lambda$ for two depths: a large $h=2$ and a shallow $h=0.2$. The $\Omega_{i}$ follow the usual trend, with the values being smaller for the shallow depths. For small values of $\lambda,-\Omega_{r}$ is almost independent of depth. However, for $\lambda>0.02$, the larger depth has the higher damping rate with a peak value that 

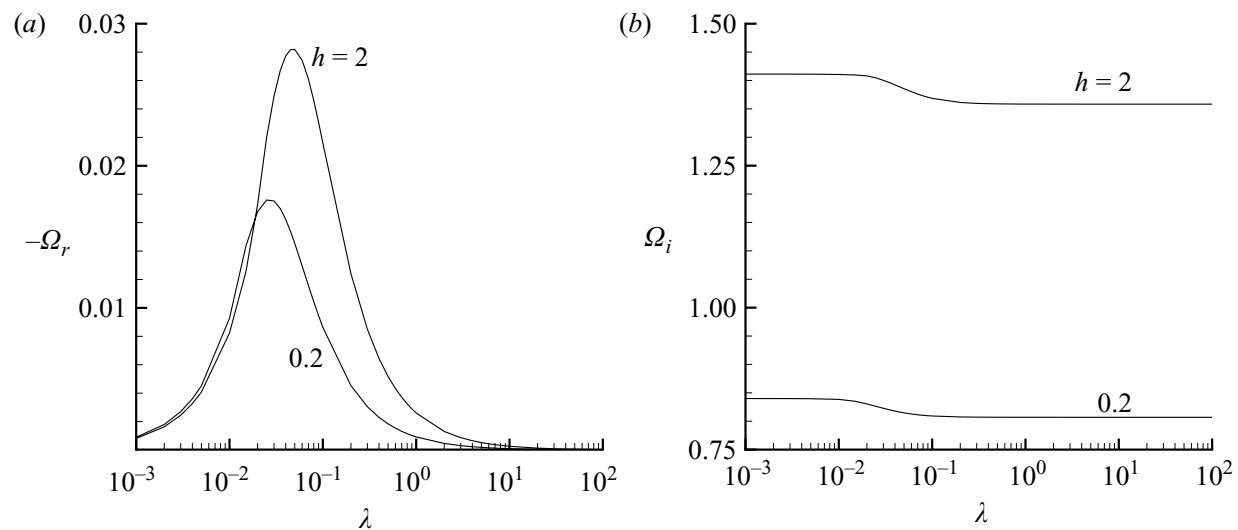

FIGURE 5. Variation of the $(a)$ damping rates and $(b)$ frequencies of the $(1,0)$ mode with liquid depth $h$. Here $B o=1000$.

\begin{tabular}{llllllll} 
& \multicolumn{7}{c}{$\theta_{c 0}$} \\
\cline { 2 - 8 }$\lambda$ & \multicolumn{1}{c}{$45^{\circ}$} & $60^{\circ}$ & $75^{\circ}$ & $90^{\circ}$ & $105^{\circ}$ & $120^{\circ}$ & $135^{\circ}$ \\
0.001 & 0.00054 & 0.00085 & 0.0011 & 0.0011 & 0.00099 & 0.00074 & 0.00043 \\
0.01 & 0.0054 & 0.0085 & 0.0116 & 0.0110 & 0.0099 & 0.0075 & 0.0043 \\
0.1 & 0.0583 & 0.0780 & 0.0864 & 0.0868 & 0.0805 & 0.0677 & 0.0469 \\
1.0 & 0.0332 & 0.0284 & 0.0266 & 0.0256 & 0.0251 & 0.0252 & 0.0274 \\
10.0 & 0.0035 & 0.0029 & 0.0027 & 0.0026 & 0.0025 & 0.0026 & 0.0029 \\
100.0 & 0.00035 & 0.00029 & 0.00027 & 0.00026 & 0.00026 & 0.00026 & 0.00029
\end{tabular}

TABLE 3. Effect of the meniscus on the damping rate $-\Omega_{r}$ of the $(1,0)$ mode for various $\lambda$. Here $B o=100, h=2$.
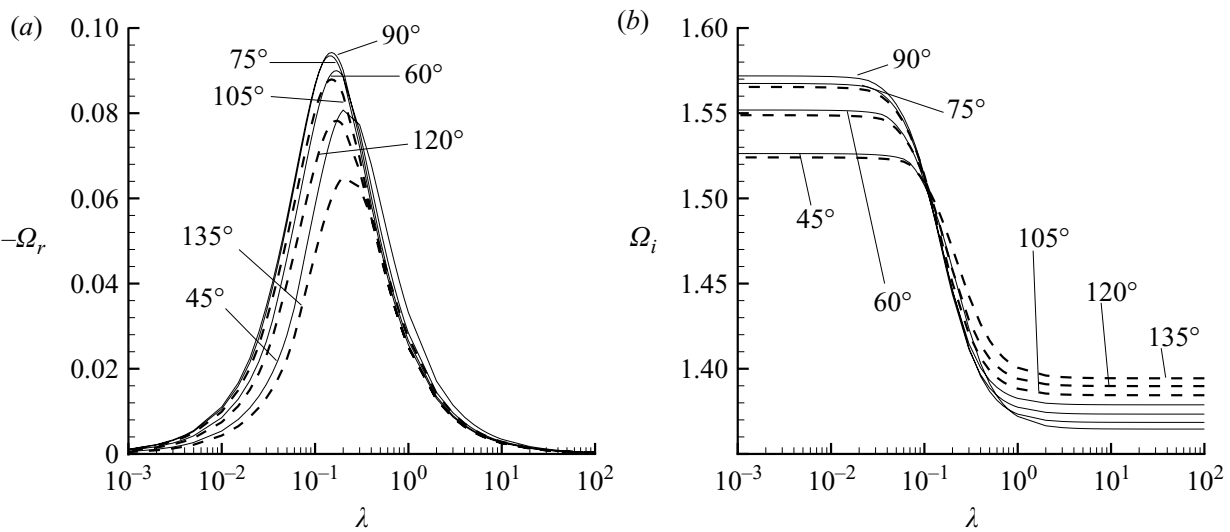

FIGURE 6. Variation of the $(a)$ damping rates and $(b)$ frequencies of the $(1,0)$ mode with capillary coefficient $\lambda$ for various contact angles. Here $h=2, B o=100$.

is almost double that of the shallow depth. This feature of capillary damping is quite different from that of viscous damping where the damping rate increases with decreasing depth.

We present the effects of the meniscus on $\Omega_{i}$ and $-\Omega_{r}$ in table 3 and figure 6 . The contact angle $\theta_{c 0}$ is varied for fixed $B o=100$ and $h=2$. For $\lambda \rightarrow 0, \Omega_{i}$ have 
a maximum around $90^{\circ}$. The distribution around the maximum is not perfectly symmetric, with the acute values being marginally bigger than the obtuse ones. For $\lambda \rightarrow \infty, \Omega_{i}$ increases monotonically with $\theta_{c 0}$. Both these findings are in accord with Nicolás (2005) for $\lambda=0$ and $\lambda=\infty$, respectively. For $\theta_{c 0}>90^{\circ}, \Omega_{i}^{p}-\Omega_{i}^{f}$ decreases. The effect of the meniscus on the damping rate is rather more interesting; values for a five-decadal range of $\lambda$ are shown in table 3 . For fixed $\theta_{c 0}$, the damping rate varies over three orders of magnitude with $\lambda$; the maximum damping rate occurs around $\lambda=0.1$ irrespective of contact angle. For $\lambda>1$, the damping rates decrease for increasing $\theta_{c 0}$ up to about $105^{\circ}$ and increase beyond that. The maximum change is of the order of $20 \%$. For $\lambda<1$, the damping rates behave exactly opposite to the above, increase for increasing $\theta_{c 0}$ up to about $90^{\circ}$ and decrease beyond that. Also, unlike for $\lambda>1$, the changes are now substantial ranging from a maximum of around $100 \%$ for the lower $\lambda=0.001$ and 0.01 to around $50 \%$ for $\lambda=0.1$. The damping rate for obtuse angles is lower than that for the corresponding acute angles. From figure 6 we see that the location of the maxima for the pairs $\left(75^{\circ}, 105^{\circ}\right),\left(60^{\circ}, 120^{\circ}\right),\left(45^{\circ}, 135^{\circ}\right)$ is almost the same. The damping rates for the case when the contact line is close to being pinned $(\lambda \rightarrow 0)$ are larger than those for the case where $\lambda \rightarrow \infty$ (free contact line). How much larger depends on the contact angle, with a smaller meniscus leading to a large increase of almost $300 \%$ and larger menisci producing only increases of the order of $50 \%$.

\subsubsection{Comparison with analytic results of Miles (1991)}

Miles (1967) estimated the capillary damping $\gamma_{L}$ as

$$
\gamma_{L}=\frac{0.451 \omega_{1}}{\sqrt{B o} \lambda},
$$

where $\omega_{1}$ is the frequency corresponding to the free-end edge condition. In deriving this estimate, Miles made the implicit assumption that the contact line velocity is that predicted by free-end edge conditions. However, this assumption is correct only for $\lambda \gg 1$ and hence (4.1) is valid only for $\lambda \gg 1$. Note in particular that (4.1) predicts an infinite damping for $\lambda=0$ whereas it is actually zero.

For $\lambda \ll 1$ and neglecting the meniscus, Miles (1991) estimated the complex frequency of the $(1,0)$ mode by a boundary layer approximation; this is given by ((4.3b), Miles 1991)

$$
\frac{\omega^{2}-\omega_{11}^{2}}{\omega_{11}^{2}}=\frac{2.84}{\sqrt{B o}(1-\mathrm{i} \gamma)} .
$$

The imaginary part of $\omega$ gives the capillary damping $\gamma_{L} \cdot \gamma$ is complex, in general, as it is obtained by scaling a velocity with the complex frequency $\omega((1.7 b)$, Miles 1991). However, in the present case where $\lambda$ has been assumed real, $\gamma$ is related to $\lambda$ by $\gamma=\lambda / \sqrt{B o}$.

Figure 7(a-d) shows Miles' analytic result (dashed lines) as well as the present numerical results (solid lines) for the $(1,0)$ mode for four Bond numbers. The damping rates by the two methods compare quite well with the location of the maximum damping rate being given, from (4.2), by $\lambda_{\max }=1 / \sqrt{B o}$. The analytic frequencies agree with the present results for $\lambda \rightarrow \infty$ while underpredicting them for $\lambda \rightarrow 0$. This was already noted by Miles (1991) where a comparison with GrahamEagle (1983) is given. We show, in table 4, both these results as well as the present result for the frequency for $\lambda=0$ and a few Bond numbers. As can be seen, the present results are in excellent agreement with Graham-Eagle (1983). Miles (1991), 

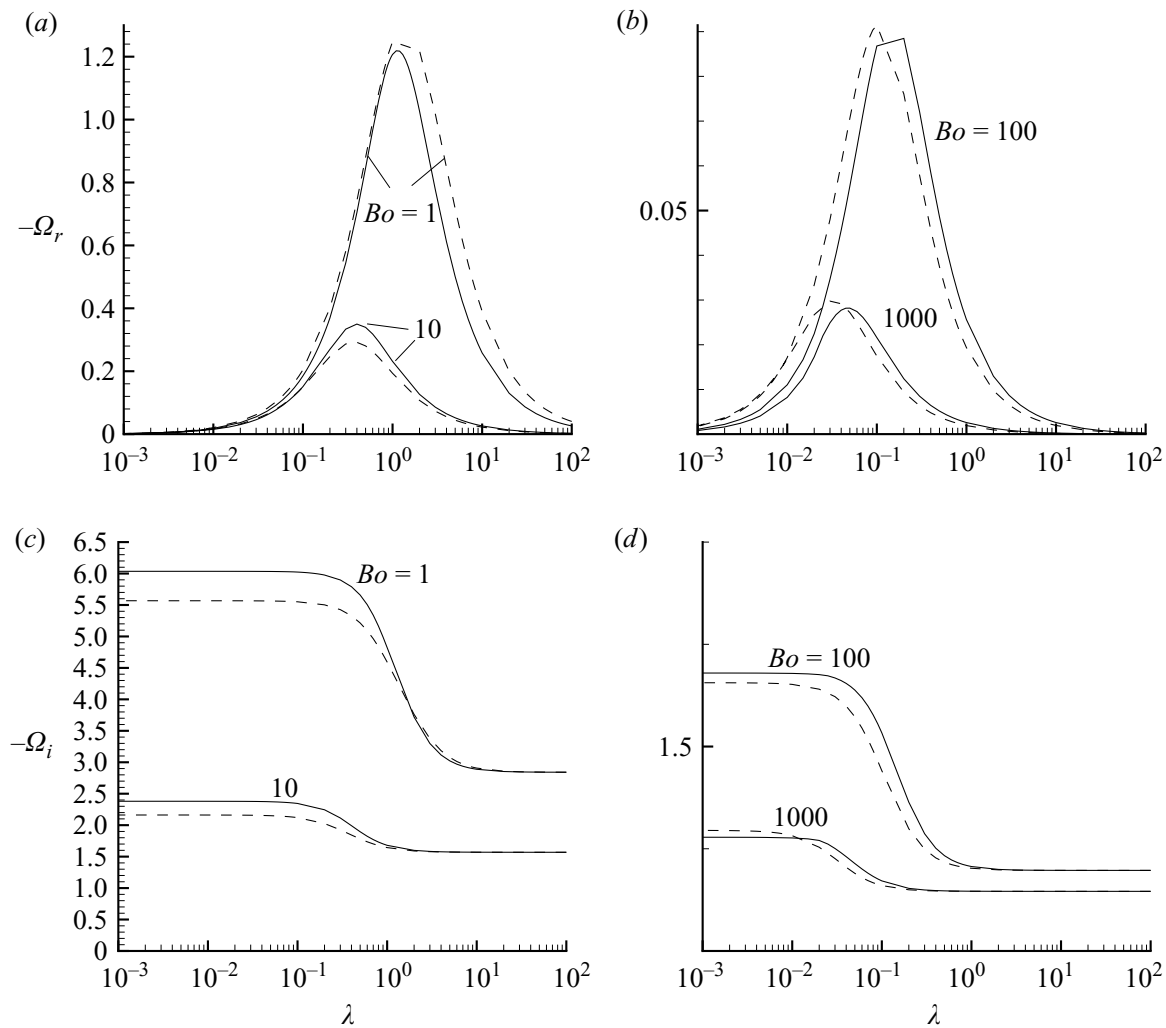

FIGURE 7. Comparison between the present numerical (solid) and analytical results of Miles (1991) (dashed). ( $a, b)$ Damping rate and $(c, d)$ frequency. Here $B o=1,10,100$ and 1000.

\begin{tabular}{rccc} 
& \multicolumn{3}{c}{$\Omega_{i}^{2} / \Omega_{1}^{2}$} \\
\cline { 2 - 4 } Bo & Present & Graham-Eagle $(1983)$ & Miles $(1991)$ \\
100 & 1.300 & 1.303 & 1.284 \\
20 & 1.829 & 1.83 & 1.64 \\
10 & 2.300 & 2.30 & 1.90 \\
5 & 2.947 & 2.95 & 2.27
\end{tabular}

TABLE 4. Comparison of the frequency of the $(1,0)$ mode for $\lambda=0$. Note that Miles' (1991) results underpredict the frequencies for all $B o$ with the discrepancy becoming worse with decreasing Bond number.

apart from the boundary layer analysis, also has an exact eigenvalue equation which he does not use to generate numbers. A solution of this equation is likely to be in much closer agreement with Graham-Eagle's and the present results.

\subsubsection{Comparison with experiments of Cocciaro et al. (1993)}

In figure 8, we present results for the experimental parameters of Cocciaro et al. (1993) who studied the characteristics of the $(1,0)$ mode in a circular cylinder. Two oscillation regimes were identified: (i) a low-amplitude regime where the contact line was pinned and a single frequency described the oscillation and (ii) a higher amplitude regime where the frequency was dependent on the oscillation amplitude 

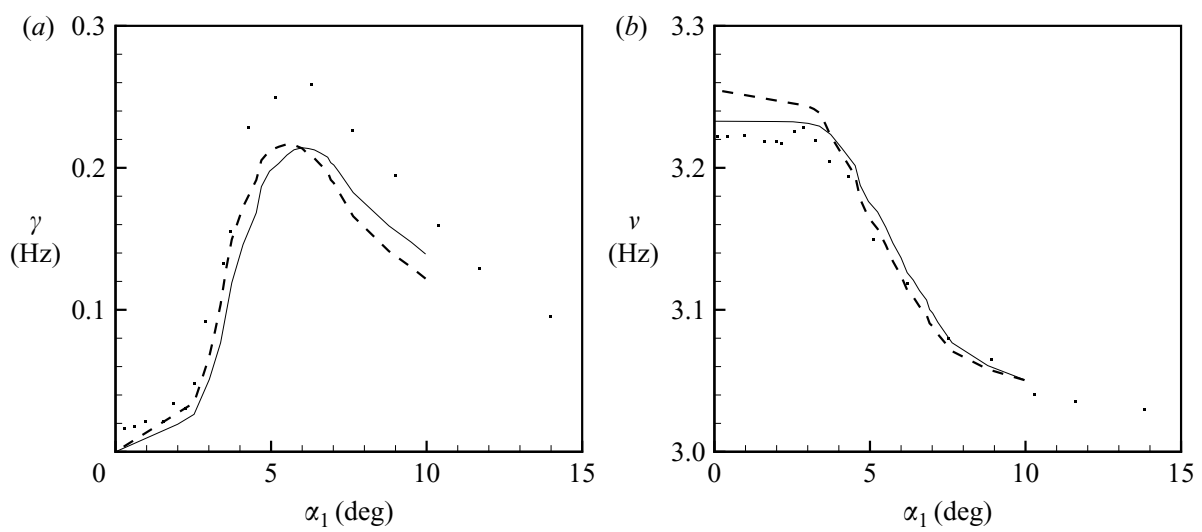

Figure 8. Variation of the $(a)$ damping rates and $(b)$ frequencies of the $(1,0)$ mode with $\lambda$. Solid lines represent present calculations which take the meniscus into account, dashed lines calculations neglecting the meniscus and the dots are experimental results from Cocciaro et al. (1993). Here $B o=346.363, h=2.578$ and $\theta_{c 0}=62^{\circ}$ are the parameters as in Cocciaro et al. (1993).

and the maximum damping rate was almost 10 times that of the first regime. Thus, Cocciaro et al. could not find a linear Hocking regime at all in their experiment; nevertheless, by making the capillary coefficient velocity-dependent and employing the wetting condition with a non-constant $\lambda$, they could reproduce some of the observed behaviour by using earlier asymptotic analyses of Miles and Hocking. These latter however do not incorporate meniscus effects.

We have computed the complex frequency for this case. Like Cocciaro et al. (1993) we also use a non-constant $\lambda$. The difference from Cocciaro et al. is that meniscus effects are also included. The present $\lambda$ is different from that defined in Cocciaro et al.; the two are related by

$$
\lambda=\lambda_{c o c c} \frac{\omega}{\sqrt{B o}} \sqrt{\frac{R}{g}}=0.078 \lambda_{c o c c},
$$

where $\omega=20.29 \mathrm{rad} \mathrm{s}^{-1}$ and $R=5.025 \mathrm{~cm}$ are experimental parameters from Cocciaro et al. (1993). The Reynolds number here is, of course, infinite as against the experimental $R e=35628.103$. Figure 8 shows the damping rate and frequency as a function of $\alpha_{1}$. The dimensional damping rate $\gamma$ and frequency $v$ are related to $-\Omega_{r}$ and $\Omega_{i}$ by $\pi \gamma=-\sqrt{g / R} \Omega_{r}$ and $2 \pi \nu=\sqrt{g / R} \Omega_{i}$, respectively. $\alpha_{1}$ is the amplitude of the first harmonic component of the tilt angle $\alpha_{f}$ of the free surface at the centre (Cocciaro et al. 1993). We relate $\lambda$ with $\alpha_{1}$ as follows. From figure 3(a) of Cocciaro et al. (1993), the oscillation amplitude $A_{c}$ of the contact line is obtained as a function of $\alpha_{1}$ and we use the same relation between $\lambda$ and $A_{c}$ as Cocciaro et al. (in (A 7)) to get

$$
\lambda=0.078 \frac{2 D \omega}{\pi} A_{c}
$$

Here, $D$ is a constant estimated, from the experiment of Cocciaro et al. (1993), to be $0.5 \pm 0.05 \mathrm{~s} \mathrm{~cm}^{-1}$. The solid line in figure 8 represents the calculation taking the meniscus into account, the dashed line is without the meniscus effects and the experimental results of Cocciaro et al. are represented by dots. The peak damping rate (figure $8 a$ ) is relatively unaffected by the meniscus; its location, however, is 


$\begin{array}{llllllllll}\alpha_{1}\left({ }^{\circ}\right) & 2.52 & 3.74 & 4.69 & 5.51 & 6.00 & 6.59 & 7.02 & 8.79 & 9.97 \\ \gamma(\mathrm{Hz}) & 0.0264 & 0.119 & 0.187 & 0.209 & 0.214 & 0.210 & 0.202 & 0.159 & 0.139 \\ \nu(\mathrm{Hz}) & 3.232 & 3.224 & 3.188 & 3.158 & 3.137 & 3.114 & 3.098 & 3.055 & 3.051\end{array}$

TABLE 5. Damping rate and frequency of the $(1,0)$ mode as a function of $\alpha_{1}$. Here $h=2.578, B o=346.363$ and $\theta_{c 0}=62^{\circ}$ as in the experiment of Cocciaro et al. (1993).

shifted to a higher value of $\alpha_{1}$ when meniscus effects are taken into account. For lower values of $\alpha_{1}$, the no-meniscus results are in closer agreement with experiment whereas the opposite is true for higher values of $\alpha_{1}$. Both theoretical curves are a little below the experimental points as the viscous contribution has not been added. According to Cocciaro et al. (1993), this is estimated to range from 12 to $18 \mathrm{mHz}$ as $\lambda$ goes from 0 to $\infty$. Thus, the viscous contribution should bring the theoretical and experimental results into much closer agreement. Also, the experimental location of the peak damping rate is in much closer agreement with the calculations that take the meniscus into account.

The influence of the meniscus is also clearly seen in the frequency (figure $8 b$ ) where the dashed theoretical curve is slightly above the experimental values as $\alpha_{1} \rightarrow 0$ because of neglect of the meniscus. The calculation which does take the meniscus into account is in closer agreement with the experimental values especially as $\alpha_{1} \rightarrow 0$, though in general the theoretical curve is slightly above the experimental values. Note that this is an inviscid calculation and an inclusion of viscous effects would induce a downward correction in the frequencies, thus bringing them into closer agreement with the experimental values. A local maximum in the frequencies, around $\alpha_{1}=4^{\circ}$, is however not captured in the computation which shows a monotonic trend. An explanation of this may require a closer examination of the viscous moving contact line. Numerical values of $\gamma$ and $v$ are given in table 5 for a few $\alpha_{1}$. The peak value of around $214 \mathrm{mHz}$ compares very well with the experimental result of around $250 \mathrm{mHz}$ (figure $9 a$ of Cocciaro et al. 1993) especially if a viscous contribution, which cannot be calculated here, of $18 \mathrm{mHz}$ (Cocciaro et al. 1993) is added.

\section{Conclusion}

We have presented a novel eigenvalue formulation to take into account the effect of a wetting condition on inviscid surface capillary-gravity waves in a circular cylinder. Though the flow is potential and the eigenvalues for the potential come from the classical zero normal velocity boundary condition, the $\eta$ eigenvalues turn out to be complex and are not known a priori. These and the complex frequency $\Omega$ are found by solving iteratively a matrix eigenvalue problem along with the Miles' wetting condition. For fixed capillary coefficient $\lambda$, the capillary damping increases with the inviscid frequency - it is thus higher for the higher modes, lower Bond numbers and larger depths. This is in accord with Miles $(1967,1991)$. The frequency behaviour is consistent with earlier published results; the pinned contact line has a higher frequency than the free one and the dependence on the contact angle is also different in the two cases.

Meniscus effects along with the Miles' condition are considered for the first time and it is shown that the effect of the meniscus on damping rate depends on the static contact angle. For hydrophobic surfaces $\left(\theta_{c 0}>90^{\circ}\right)$, a meniscus seems to 
always reduce the damping irrespective of the capillary coefficient. For hydrophilic surfaces $\left(\theta_{c 0}<90^{\circ}\right)$, a meniscus can increase or reduce the damping depending on the capillary coefficient $\lambda$. Calculations show that for $\lambda \rightarrow 0$, a smaller contact angle (larger meniscus) leads to a decreased damping rate whereas for $\lambda \rightarrow \infty$, the opposite holds and a smaller contact angle leads to an increased damping rate. This is, to our knowledge, a new result and quite different from how a meniscus contributes to the viscous damping where it could either reduce (for obtuse angles) or increase (for acute ones) the damping (Kidambi 2009). Also, the location of peak damping shifts to higher $\lambda$ both for acute and obtuse angles; the location seems to be the same for supplementary angles. The meniscus effect on the frequency is well known for the pinned case $\lambda=0$ (Nicolás 2005; Shankar 2007); it is here shown to be true for non-zero $\lambda$ as well. Interesting differences between the cases of acute and obtuse contact angles are seen.

The consequence of taking the meniscus into account has been examined in a situation for which experimental results are available (Cocciaro et al. 1993). While the phenomenon is essentially nonlinear and can only be captured in an ad hoc manner by a linear analysis, the inclusion of the meniscus shows that certain details like the location of peak damping are captured more accurately than if the meniscus was not included. For the experimental parameters considered, where $B o$ is fairly high, no significant difference in the peak damping value, between the meniscus and no-meniscus results, was seen. However, as table 3 and figure 6 show, the meniscus could make significant contributions for lower $B o$; a $\theta_{c 0}=45^{\circ}$ could reduce the damping rate by as much as about 0.03 for a $B o=100$ and $\lambda=0.1$, compared to the no-meniscus case. This would translate to a dimensional $\gamma \approx 133 \mathrm{mHz}$ (using the same cylinder of Cocciaro et al. 1993), which is almost $35 \%$ of the peak value of $386 \mathrm{mHz}$ that occurs for $\theta_{c 0}=90^{\circ}$. This not only is a significant contribution by the meniscus to the inviscid damping but is also an order of magnitude higher than the viscous contribution which is estimated to be, using (2.11a) of Cocciaro et al. and a capillary length $\lambda_{c}=5 \mathrm{~mm}$ (corresponding to $B o=100$ ), of the order of $19-33 \mathrm{mHz}$.

Hocking (1987) indicated two directions in which the work might be continued, by including the effects of a finite depth and a meniscus. This work is a step towards those directions, even if only for the inviscid case.

I thank the anonymous referees for their valuable comments which have helped me to correct some errors and improve the presentation considerably. The work is funded by the Aeronautical Research \& Development Board, India, under Project No. 1031340.

\section{Appendix A}

A convenient way to solve $(2.2 a)$ is to write $\eta_{s}$ as

$$
\eta_{s}(r)=\sum_{n=1}^{N} u_{m}\left[\cos k_{m} r+(-1)^{m} \frac{k_{m}}{2}\left(1-r^{2}\right)\right]-\frac{\cot \theta_{c 0}}{2}\left(1-r^{2}\right),
$$

where $k_{m}=(2 m-1) \pi / 2$ and the coefficients $u_{m}$ are obtained by a least-squares error minimization procedure. Note that the boundary conditions are satisfied by the assumed form for $\eta_{s}(r)$. 
The minimization procedure consists of the following steps:

(a) First write $(2.2 a)$ as

$$
\eta_{s}(r)-\frac{1}{B o}\left[\eta_{s}^{\prime \prime}+\frac{\eta_{s}^{\prime}}{r}\right]-\zeta=\frac{1}{B o}\left[\eta_{s}^{\prime \prime}\left(\frac{1}{\left(1+\eta_{s}^{\prime 2}\right)^{3 / 2}}-1\right)+\frac{\eta_{s}^{\prime}}{r}\left(\frac{1}{\left(1+\eta_{s}^{\prime 2}\right)^{1 / 2}}-1\right)\right]
$$

and consider $N$ equally spaced points $r_{i}=i /(N-1), i=0,1, \ldots, N$ over which the error in the above equation will be minimized.

(b) Define the error at the $i$ th point $e_{i}$ as

$$
e_{i}=\eta_{s}(i)-\frac{1}{B o}\left[\eta_{s}^{\prime \prime}(i)+\frac{\eta_{s}^{\prime}(i)}{r_{i}}\right]-\zeta-r h s(i)
$$

where $r h s(i)$ is the right-hand side of the equation in $(a)$ evaluated at the $i$ th point.

(c) Consider the total squared error $E_{t}$ defined as

$$
E_{t}=\sum_{i=1}^{N} e_{i}^{2}
$$

The coefficients $u_{m}$ are obtained by minimizing $E_{t}$ with respect to $u_{m}$, i.e. by setting $\partial E_{t} / \partial u_{m}=0, m=1, \ldots, N$ and solving the resulting system of linear equations.

(d) These $u_{m}$ are now used to calculate $\eta_{i}$, its derivatives and $e_{i}$. If the maximum error is less than an assumed tolerance $\epsilon$, the calculation is done.

(e) If not, use $\eta_{i}$ and its derivatives obtained above to calculate $r h s(i)$. Repeat (b)-(d) to convergence.

The calculation starts with the initial guess $r h s(i)=0$. Finally, the parameter $\zeta$ is obtained from

$$
\zeta=\frac{1}{B o}\left[\left(\sum_{n=1}^{N} u_{n} k_{n}(-1)^{n}-\cot \theta_{c 0}\right) \sin ^{3} \theta_{c 0}-\cos \theta_{c 0}\right] .
$$

\section{REFERENCES}

Ablett, R. 1923 An investigation of the angle of contact between paraffin wax and water. Phil. Mag. 46, 244-256.

Benjamin, T. B. \& Scott, J. C. 1979 Gravity-capillary waves with edge constraints. J. Fluid Mech. 92, 241-267.

Case, K. M. \& Parkinson, W. C. 1957 Damping of surface waves in an incompressible liquid. J. Fluid Mech. 2, 172-184.

Cocciaro, B., FaetTi, S. \& Festa, C. 1993 Experimental investigation of capillarity effects on surface gravity waves: non-wetting boundary conditions. J. Fluid Mech. 246, 43-66.

Graham-Eagle, J. 1983 A new method for calculating eigenvalues with applications to gravitycapillary waves with edge constraints. Math. Proc. Camb. Phil. Soc. 94, 553-564.

Henderson, D. M. \& Miles, J. W. 1994 Surface-wave damping in a circular cylinder with a fixed contact line. J. Fluid Mech. 275, 285-299.

Hocking, L. M. 1987 The damping of capillary-gravity waves at a rigid boundary. J. Fluid Mech. 179, 253-266.

Howell, D. R., Buhrow, B., Heath, T., McKenna, C., Hwang, W. \& Schatz, M. F. 2000 Measurements of surface-wave damping in a container. Phys. Fluids 12 (2), 322-326.

Jiang, L., Perlin, M. \& Schultz, W. W. 2004 Contact-line dynamics and damping for oscillating free surface flows. Phys. Fluids 16 (3), 748-758.

Johnson, R. S. 1997 A Modern Introduction to the Mathematical Theory of Water Waves. Cambridge University Press.

Keulegan, G. H. 1959 Energy dissipation in standing waves in rectangular basins. J. Fluid Mech. 6, 33-50. 
KidAmbi, R. 2009 Meniscus effects on the frequency and damping of capillary-gravity waves in a brimful circular cylinder. Wave Motion 46, 144-154, doi:10.1016/j.wavemoti.2008.10.001.

Lamb, H. 1895 Hydrodynamics, 2nd ed. Cambridge University Press.

Martel, C., Nicolás, J. A. \& Vega, J. M. 1998 Surface-wave damping in a brimful circular cylinder. J. Fluid Mech. 360, 213-228.

Miles, J. W. 1967 Surface-wave damping in closed basins. Proc. R. Soc. Lond. A 297, 459-475.

Miles, J. W. 1990 Capillary-viscous forcing of surface waves. J. Fluid Mech. 219, 635-646.

Miles, J. W. 1991 The capillary boundary layer for standing waves. J. Fluid Mech. 222, 197-205.

Nicolás, J. A. 2002 The viscous damping of capillary-gravity waves in a brimful circular cylinder. Phys. Fluids 14, 1910-1919.

Nicolás, J. A. 2005 Effects of static contact angles on inviscid capillary-gravity waves. Phys. Fluids 17, 022101.

Shankar, P. N. 2007 Frequencies of gravity-capillary waves on highly curved interfaces with edge constraints. Fluid Dyn. Res. 39, 457-474.

VAN Dorn, W. G. 1966 Boundary dissipation of oscillatory waves. J. Fluid Mech. 24, 769-779. 